\title{
Libros académicos en la Nueva España
}

\author{
Rosa-María Fernández-de-Zamora
}

\section{RESUMEN}

Mucho se podría escribir sobre los libros que se leyeron para las actividades académicas de la Real Universidad y otras instituciones educativas como el Colegio de Minería, la Academia de San Carlos y otros colegios entre los que destacan los de los jesuitas. Sin embargo, en este artículo se desea resaltar los libros académicos impresos tanto en Europa como en México que puedan ser reconocidos, porque la manera en que están citados dificulta la tarea. Igualmente se ha tratado de identificar los títulos y los autores especialmente en el catálogo de la Biblioteca Nacional de México porque es la institución que recibió la mayor herencia bibliográfica de la época colonial, pero también en otras bibliotecas. Se respetó la ortografía original de los textos mencionados y éstos son acompañados de ilustraciones de sus portadas.

Palabras clave: libros académicos, Real y Pontificia Universidad de México, universidad, libros de texto en Nueva España, México. 


\section{Livros acadêmicos na Nova Espanha}

\section{RESUMO}

Muito se poderia escrever sobre os livros que se leram para as atividades acadêmicas da Real Universidad e outras instituições educativas como o Colegio de Minería, a Academia de San Carlos e outros colégios entre os quais destacam os dos jesuítas. Porém, neste artigo se deseja ressaltar os livros acadêmicos impressos tanto na Europa como no México que possam ser reconhecidos, porque a maneira em que estão citados dificulta a tarefa. Igualmente se tem tratado de identificar os títulos e os autores especialmente no catálogo da Biblioteca Nacional de México porque é a instituição que recebeu a maior herança bibliográfica da época colonial, mas também em outras bibliotecas. Se respeitou a ortografia original dos textos mencionados e estes são acompanhados de ilustrações de suas portadas.

Palavras chave: livros acadêmicos, Real e Pontificia Universidad de México, universidade, livros de texto na Nueva Espanha, México.

\section{Academic books in New Spain}

\section{ABSTRACT}

Much could be written about the books that were read for the academic activities of the Royal University of Mexico and other educational institutions such as the Colegio de Minería, the Academia de San Carlos and other schools among which those of the Jesuits stand out. However, in this article the authors wish to highlight the academic books printed in Europe as well as in Mexico that can be recognized as part of other discourses, because the way they are cited makes the task difficult. We have tried to identify titles and authors especially in the catalog of the National Library of Mexico because it is the institution that received the largest bibliographic heritage of the colonial period, but also in other libraries. The original spelling of the mentioned texts was respected and they are accompanied by illustrations of their covers.

Keywords: academic books, Real y Pontificia Universidad de México, university, textbooks in New Spain, Mexico.

Recepción: 18/10/19. Aprobación: 30/07/20. 


\section{Introducción}

La Real Universidad fue fundada por Carlos V el 21 de septiembre de 1551, a través de su hijo el príncipe Felipe, la ceremonia de inauguración fue en enero de 1553 y el 3 de junio de ese año comenzaron las lecciones con la cátedra de retórica de Francisco Cervantes de Salazar. Fue la primera universidad laica de América pues como asienta Pérez (1970: 18), "La universidad de México fue la primera universidad seglar y pública del continente americano creada con sentido integracionista para 'los naturales' y los hijos de los españoles. Su vida fue fecunda e ininterrumpida hasta que la estructura social en que había nacido se modificó sustancialmente". Fue una universidad laica por casi 50 años, sus cursos no se dieron en un estudio del convento de los dominicos como sucedió en las universidades de San Marcos y de Santo Domingo. La universidad tenía su sede propia y contaba con un presupuesto otorgado por el rey para pagar a sus maestros y cubrir otros gastos. La Universidad fue reconocida por el papa hasta finales de siglo cuando se convirtió en Real y Pontificia Universidad. Fue la primera universidad del continente que abrió sus cursos y poco a poco se convirtió en la universidad más importante del Nuevo Mundo por sus contribuciones académicas, favorecida por el ambiente cultural y tecnológico que se vivía en México ya que se disponía de la imprenta desde 1539. Fue la primera universidad fuera de Europa que utilizó la imprenta para apoyar sus actividades y de la única que se conservan tesis para optar por un título o grado académico en la Universidad.

La información disponible sobre el funcionamiento y la organización de la Real Universidad es poco clara y en ocasiones contradictoria, como veremos en el desarrollo de este escrito.

La Universidad abrió sus cursos con tres facultades mayores, las de Teología, Cánones y Leyes y una menor, de Artes, además de la cátedra de Medicina, posteriormente facultad. Algunos autores mencionan que funcionó con cinco facultades, sin mencionar las características anteriores. Otorgaba el grado menor de bachiller y los grados mayores de licenciado, maestro y doctor.

La Universidad comenzó con ocho o diez cátedras, a mediados del siglo XVII contaba con 23, incluyendo dos de las lenguas mexicanas náhuatl y otomí y al finalizar el siglo XVIII se impartían 28. El método habitual de enseñanza era la lectio, o explicación de un texto de allí el nombre de lectores que también se daba a los catedráticos porque básicamente leían los textos señalados por el plan de estudios seguidos de comentarios. Los catedráticos eran designados por el virrey, la audiencia y el claustro. Eran casi siempre frailes dominicos o agustinos, pero igualmente hubo muchos seglares. La lengua en que se estudiaba, se leía, se escribía y se dialogaba en el medio académico era el latín aunque también se usó el castellano.

Los estudiantes debían "legere, repetere et disputare", es decir debían aplicarse a un sistema de estudio basado en la memorización. Leer, repetir y discutir expresan con claridad y sencillez no sólo la concepción medieval de generación del conocimiento a partir de los textos sino también el sentido genérico de la escritura: hecha para ser leída, pero también, en cuanto tal, sostén de la memoria y que permite preservar y repetir la información.

En ocasiones catedráticos eminentes redactaban sus propios textos, como fueron los casos en la Nueva España, de fray Alonso de la Veracruz y de Francisco Cervantes de Salazar en el siglo XVI y José Ignacio Bartolache en el siglo XVIII.

Los libros académicos que se han podido identificar analizando los Estatutos de Cerralvo, las Constituciones de Palafox y otros textos relacionados con la Real Universidad se mencionarán a continuación.

\section{Libros académicos por facultad Facultad de Artes}

En esta facultad menor se enseñaba filosofía y lógica, Fray Alonso de la Veracruz, como maestro del primer curso de filosofía en la Real Universidad, 
escribió y publicó una obra de lógica formal, la $R e^{-}$ cognitio Summularum y otra de lógica material, la Dialectica Resolutio. En 1557 publicó la Physica Speculatio que trata de filosofía natural.

La Recognitio Summularum y la Dialectica Resolutio fueron impresas en México por Juan Pablos en 1554. La Dialectica tiene una dedicatoria de Fray Alonso a la Universidad y un prólogo de Francisco Cervantes de Salazar. La Physica Speculatio - Juan Pablos,
1557- es considerado el primer libro científico impreso en América. Los tres libros de Alonso de la Veracruz fueron textos académicos en la Real Universidad y también en la Universidad de Salamanca e impresos en esa ciudad varias veces.

La Dialectica, junto con la Recognitio Summularum y los Dialogos Latinos de Cervantes de Salazar constituyen los primeros textos universitarios publicados en México y en América.

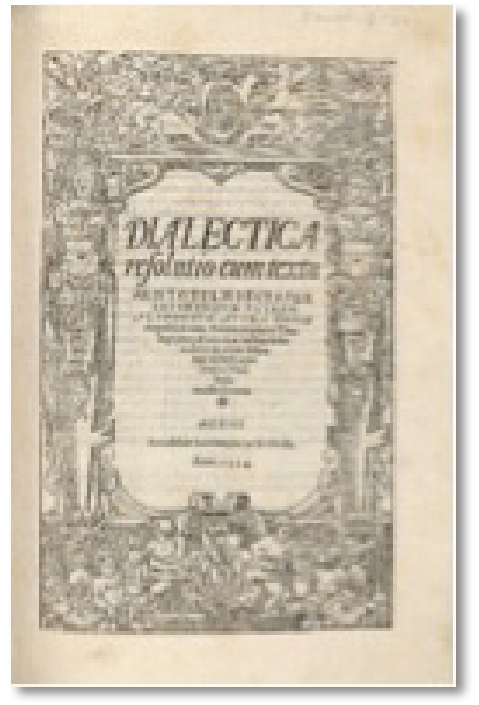

Fray Alonso de la Veracruz, 1507-1584

Dialectica Resolutio, 1554

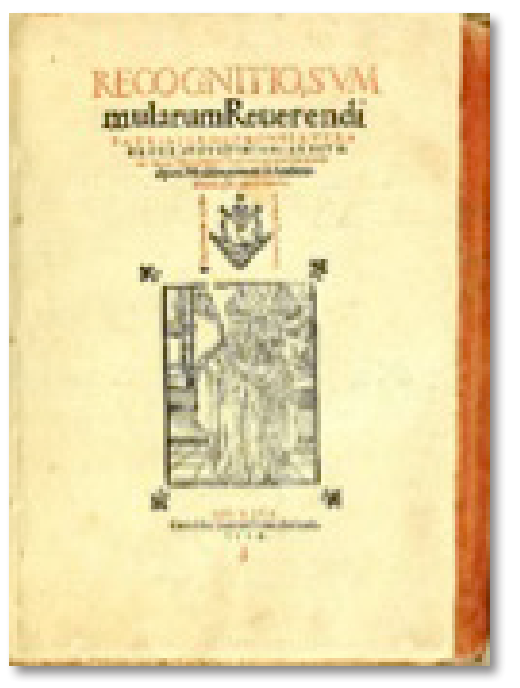

Fray Alonso de la Veracruz 1507-1584

Recognitio Sumularum, 1554

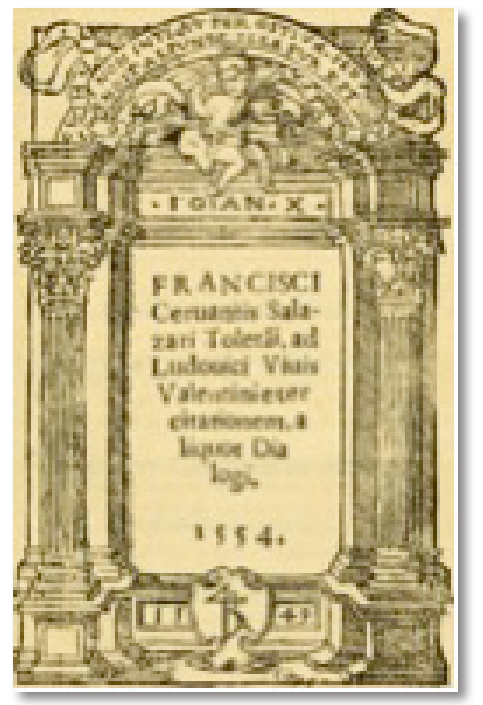

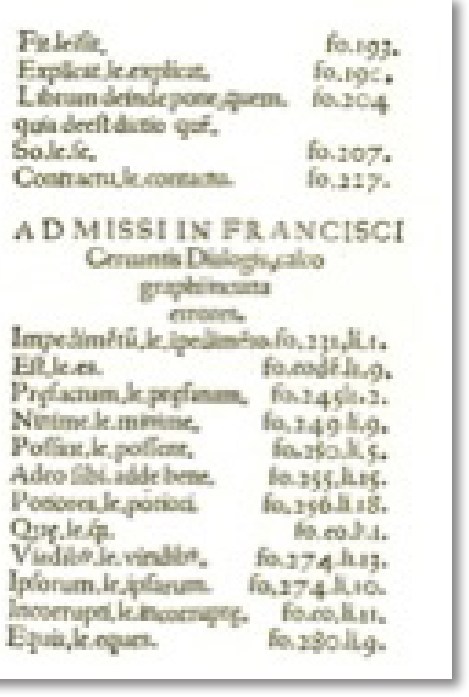

Francisco Cervantes de Salazar, 1512-1575

Dialogos latinos, 1554 
De acuerdo con los Estatutos de Cerralvo (1626), que en su Titulo 15 dice: "De lo que han de leer los catedráticos de Artes" (Proyecto..., 1991: 85), se mencionan las lecturas de los tres años que duraba el curso. En el primer año se tenían que leer las Súmulas de Villalpando y la Lógica por el maestro Domingo de Sotto. Las Súmulas de Villalpando no pudieron ser identificadas, ¿era Juan Bautista Villalpando, 15521608? La única edición de las Súmulas que se encuentra en la Biblioteca Nacional ${ }^{1}$ es de Domingo de Soto (1494-1566); Summulae, Salmanticae: In aedibus Dominici a Portonariis, 1575 (RFO 230 SOT.s. 1575, Biblioteca Nacional de México (BN))

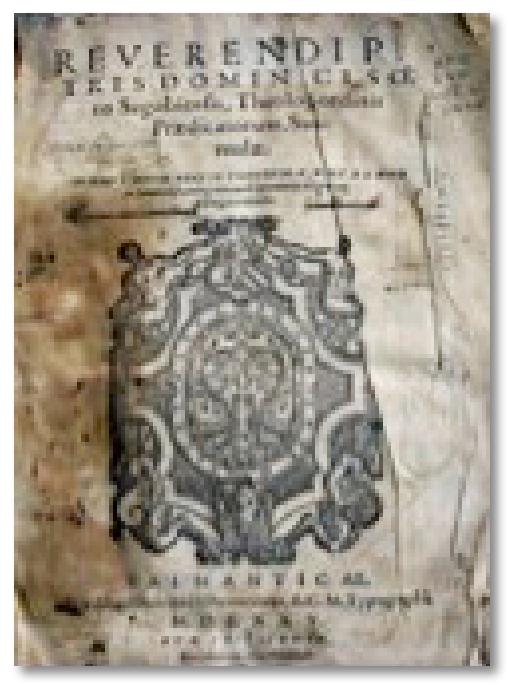

Domingo de Soto, 1494-1566 Summulae, 1575

En esto coincide Mauricio Beuchot (1987: 17) al afirmar que el primer libro de texto utilizado en la Real Universidad para la enseñanza de la filosofía en 1553, fue el de las Súmulas de Soto, para lo cual se utilizó la segunda edición española de 1543, no localizada, y las que siguieron como la ya mencionada. La Lógica de Domingo de Soto que se localizó es la impresa en Madrid en 1673, pero no se encuentra en ninguna biblioteca mexicana: Domingo de Soto
(1673), Commentaria in Aristotelis Logicam: ex doctrina sapientissimi magistri Fratris Dominici de Soto, Ordinis Predicatorum desumpta Hanc quartam editionem nec non totam Dialecticam, et Philosophiam, Sacra Deipara, nec non santissimo eiusde Rosario, dicat, et consecrat, humilis seruus F. Petrus Diaz de Cossio, eiusdem Ordinis Pradicator, Matriti; Ex Typographia Iosephi Fernandez de Buendia.

"El tercer año...se leerán los Físicos del mesmo maestro Soto" (Proyecto..., 1991: 86), la Biblioteca Nacional no tiene esta obra, pero si las siguientes sobre los Fisicos de Aristóteles:

- Johanes Canonicus Dertusiensis. Quaestiones super octo libri physicarum Aristotelis. Padue: Bonus Gallus, 1475. (1475-4-25 JOH.q., BN).

- Egidio Colonna O. S. A., Arz. M. (1316), Commentaria in octo libros physicorum Aristotelis/fratris Egidi Romani ordinis Eremitarum diui augustini..., Patauii, Hieronimi durantis 1493 (15 de octubre) (1493-10-15 COL.d., BN).

- Antonio Rubio [1548-1615] (1615), Commentarii in octo libros. Aristotelis De physico avdito una cum dvbiis et questionibvs hac tempestate agitari solitis, Compluti, Ex oficina Iusti Sanches Cresho, 1605 (RFO 186 RUB.c.1605, BN).

"E habiendo concluido con los Fisicos con la brevedad dicha, pasarán a leer los libros De jeneratione Et corruptione, e los De Anima aprovechándose de los espositores que les paresiere..." (ibid.: 86). Título 16. "Lo que han de leer los catedráticos de rretórica e gramática [...] la una hora de Laurencio Vala y la otra de un poeta o estoriador qual el rrector lo signare ad vota audientium"; la obra de Vala es:

- Lorenzo Valla [1406-1457] (1566), Elegantiarvm latinae lingvae libri sex [...] Lugduni: Apud Antonivm, 1566 (RFO.808 VAL.e.1566, BN).

\footnotetext{
${ }^{1}$ Agradezco a la Biblioteca Nacional de México su apoyo para ilustrar este artículo.
} 

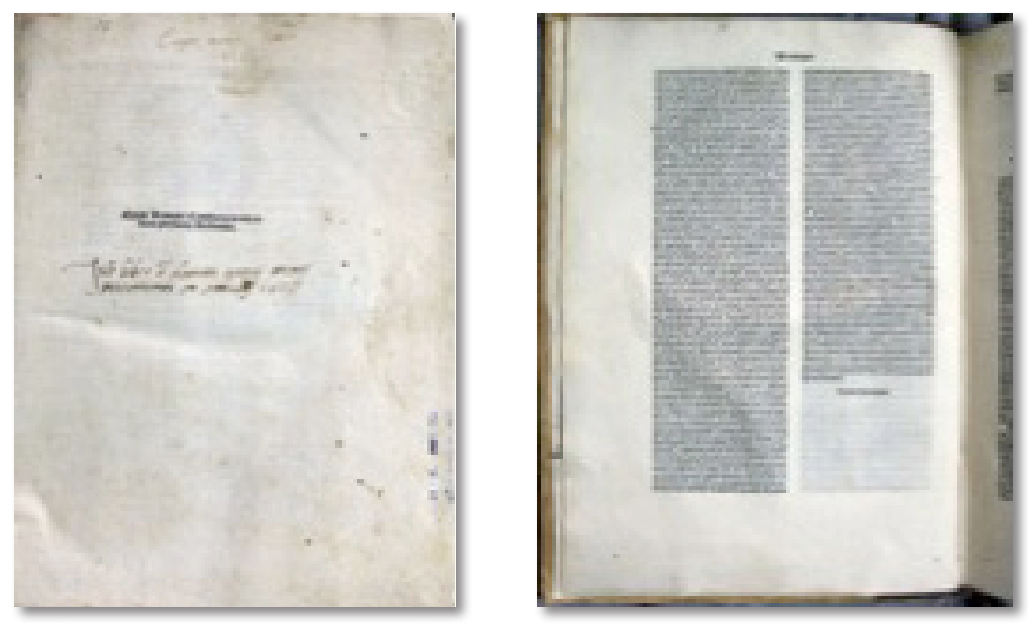

Egidio Colonna,

1316

Commentaria in octo libros Physicorum Aristotelis...

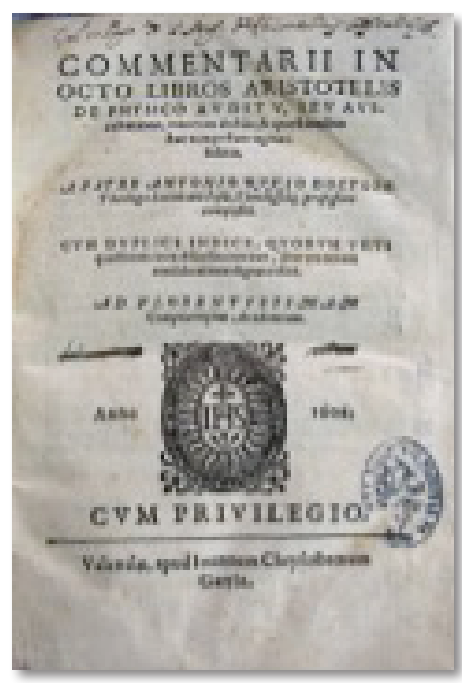

Antonio Rubio

1548-1615

Commentari in octo libros

Aristotelis, 1605

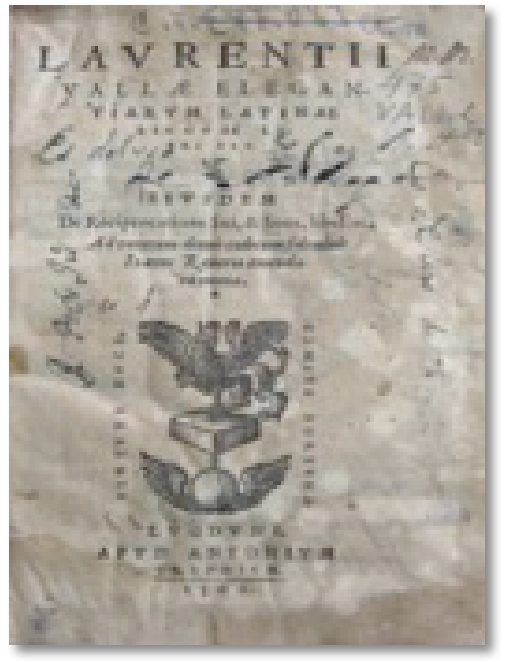

Fray Alonso de la Veracruz, 1507-1584

Recognitio Sumularum, 1554

\section{Facultad de Derecho Canónico}

Título 9 (Estatutos de Carralvo) "De lo que an de leer los catedráticos de cánones de la rreal universidad [...] desde el proemio de Gregorio 9 e primero libro de las decretales, el texto De electione..."

La enseñanza de este derecho estuvo basada en las colecciones de decretos integrados en el Corpus Iures Canonici, que son las cartas de los Papas escritas entre los siglos IV al XV, por medio de las que comunicaban sus decisiones referentes a cuestiones disciplinares; son parte del Derecho canónico.
Cuatro colecciones de decretos, junto con las decretales de Gregorio IX y el Decreto de Graciano, constituyen a partir del siglo XVI el Corpus Iuris $\mathrm{Ca}^{-}$ nonici: las colecciones de 1298, "Liber Sextus", las de 1314 "Extravagantes Clementinas", las "Decretales extravagantes" de Juan xxii (1316-34) y las "Extravagantes comunes", surgidas de 1294 a 1484.

Existen en la Biblioteca Nacional las siguientes obras:

- Iglesia Católica [Corpus Juris Canonic] (1671), 
Corpus joris canonici: in tres partes distinctum; glossis diversoruvm ilustratom/, Lvgduni, Sumptibus Ioannis Antonii Hvgvetan, Gvlielmi Barbier.

- Iglesia Católica Papa [1316-1334: Juan XXII] (1539), Extravagantes Ioannis XXII: mendis ómnibus purgatae, Lvgduni, Apud Hugonem a Porta.

- Iglesia Católica Papa [1294-1303: Bonofacio VIII] (1588), Liber sextus Decretalium D. Bonifacii Papae VIII. Clementis Papae V. Constitutiones. Extravagantes tum vigente D. Ioannis Papae XXII tum comunes, Augustae Taurinorum, s.n. 1588.

El catedrático de decreto, "El primer año comenzará el principio de las distinciones y proseguirá en esta manera: desde San Lucas a navidad leerá e acavará toda, e la Electiones" (Proyecto..., 1991: 77).

- Pierre Gregoire [1540-1597] (1592), De electione in re beneficiaria brevis et ordinata explicatio, Lvgdvni, Apud Ioan Pillehotte (RFO 262.9 GRE.c. 1594, BN).

Título 10. "De lo que a de leer el catedrático Sexto que añade en esta Univercidad". Tenía que leer dos años de Título y una de Clementinas. Las Clementinas eran las colecciones de derecho canónico publicadas por el Papa Juan XXII en 1347, comprendían los cánones del Concilio General de Viena y las decretales de Clemente V.

En la Biblioteca Nacional se encuentra, entre otras, la bella obra:

- Iglesia Católica Papa [1305-1314: Clemens V] (1554), Clementis Qvinti Constitutiones quas volgo Clementinas vocant/Aegidio Perrino fideliter emendatae, glossis, argumentis, annotationibus, iurispatronatus arbore, \& Ioannis Imolae, Ancharani, Zabarellae, Barbatiae, Panormitani, aliorímque doctorum commentaris elucidiores redditae, Lvgdvni, Ad Salamandrae, apud Sennetonios Frates (RFO 94-43937, BN).

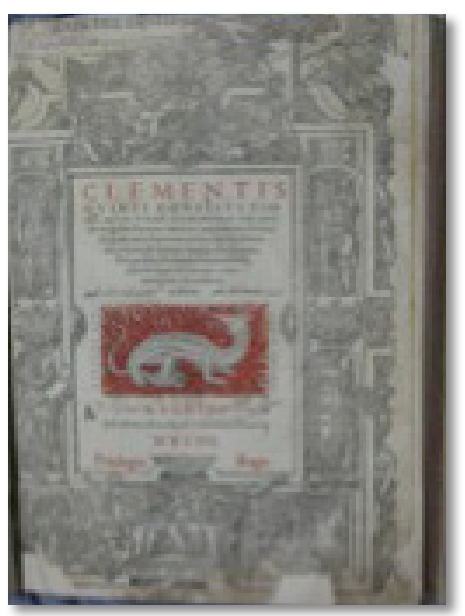

Clementis Qvinti Constitutiones, 1554

\section{Facultad de Leyes}

"El catedrático de prima de leies leerá el primero año de Digesto Biejo y el segundo del Esforsado y el tercer de Digesto nuevo" (Proyecto..., 1991: 77) relacionados con el derecho romano, el Copus Iuris Civilis, reunión de leyes romanas por orden de Justiniano.

El Digesto viejo fue publicado en el año 1529 y el Digesto Nuevo o Códice de Justiniano publicado en 1534; surgieron entonces las Pandectas, la Instituta y las Novellae. En la Biblioteca Nacional se encuentran las tres siguientes obras de Baldo degli Ubaldi (1327?-1400), que seguramente fueron textos utilizados en la Real Universidad:

- Lectura super secunda parte Digesti veteris, domini Baldi de Perusio in ciuitate, Uenetiarum, Andream de Asula, 1495 (29 de julio) (1493-10-16 BAL.d., BN).

- Lectura super Digesto Novo, Venetijs, Adream Terresanis de Asula, 1495 (18 de agosto) (1496-818 BAL.I., BN).

- Lectura super prima parte Digesti veteris, Venetijs, Andream de Thorresanis de Asula, 1493 (16 de octubre) (1493-10-16 BAL. d., BN). 
Se señalan las lecturas para el segundo y "tersero" año también relacionadas con los Digestos. En el quarto año se leerá "del título De Petitione hereditatis, elijiendo de los títulos más capitales e famosos..." (ibid.) obra no identificada. La lectura para el quinto año será De fideicomiscis:

- Marco Antonio Peregrino (1595), De fideicomissis proesertim universalibus, tractatus frequetissimvs, ómnibus tam in scholis, quam in causarum foro versantibus admodum utilis, ac pernecessarius, Venetii, Rubertum Meiettum (RFO 94-42107, BN).

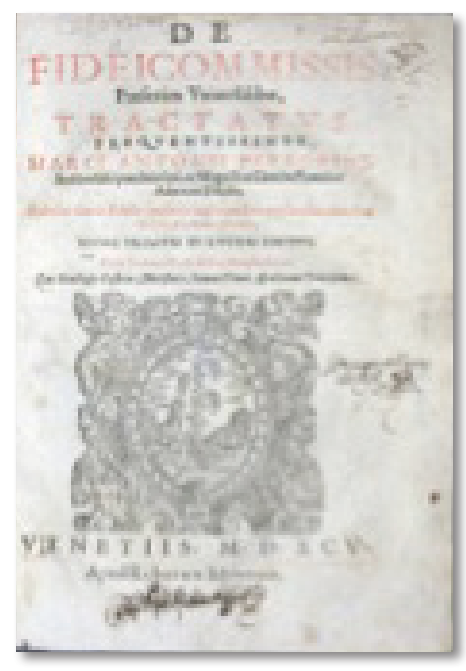

Marco Antonio Peregrino, De fideicomissis, 1595

El catedrático de instituta, "El primer año leerá, del libro primero de Ynstituta, del título De patria postestate [...] Seguno año leerá el título De rrerum devisione [...]" (ibid.) y así hasta el quinto año.

Se consideró que todos eran textos teóricos que no tenían que ver con la vida práctica, por eso en 1789 el virrey Revillagigedo propuso incorporar el derecho real al currículum de la Facultad de Leyes a través del texto de Eusebio de Ventura Beleña, Institutta Civilis Hispano Indiana, pero no fue aceptado. En 1803 el arzobispo Lizana sugirió una cátedra de eclesiástica siguiendo la obra de Thomassino Instituta Canonica (Ramírez e Hidalgo, 2001: 73). De Ventura Beleña se encuentran en la Biblioteca Nacional:
- Eusebio Buenaventura Beleña, 1736-1794 (1787), Copias a la letra ofrecidas en el primer tomo de la recopilación sumaria de todos los autos acordados de la real audiencia y la del crimen de esta, N.E. y provincias de su superior gobierno: de varias Reales Cedulas y Ordenes que, después de publicada la recopilación de Indias, han podido recogerse, asi de las dirigidas a la misma audiencia ó gobierno, como de algunas otras que por sus notables decisiones convendrá no ignorar/ por el doctor Don Eusebio Bentura Beleña, México, Félipe de Zúñiga y Ontiveros (rfo 93-31480 rfo 94-40969, BN)

- Eusebio Buenaventura Beleña, 1736-1794 (1787), Recopilación sumaria de todos los autos acordados de la Real Audiencia y Sala del Crimen de esta Nueva España, y providencias de su superior gobierno; de varias reales cédulas y órdenes que después de publicada la Recopilación de Indias han podido recogerse asi de las dirigidas á la misma Audiencia ó gobierno, como de algunas otras que por sus notables decisiones convendrá no ignorar, México, Don Felipe de Zúñiga y Ontiveros, calle del Espíritu Santo (G972.02 BEL.r., BN)

\section{Facultad de Teología}

Título 12, "De lo que an de leer los catedráticos de prima e vísperas de teulogia e biblia. En la catedra de teología de prima e vísperas, se an de leer los quatro libros de las sentencias del Maestro, como manda la constitución, desta manera que se lean las partes de santo Tomás..." (ibid.). El Maestro era Pedro Lombardo (1100-1160) teólogo y autor del libro básico para la enseñanza de la teología en los siglos XII a XVI, el Libro de las sentencias. La Biblioteca Nacional de México no tiene ningún ejemplar de este libro, en la Biblioteca Nacional de España se encuentran éstos:

- (1553), Petri Lombardi espiscopi parisiensis sententiarum libri IIII..., Parisiis, Apud Hieronymus et DionysiM DE Marnes fratres. 
- (1757), Petri Lombardi novariensis Episcopi parisiensis, Sentensiarum libri quatuor... Antuerpiae, Sumptibus Marci-Michael Bousquet 6 Socior.

De Santo Tomás de Aquino, la Biblioteca Nacional de México conserva 63 obras impresas en el siglo XVI, de estas se pueden mencionar:

- Tomás de Aquino, Santo [1225?-1274] (1562), Sommae sacrae theologiae sancti Thomae Aqvinatis, Lvgdvni, Apud haeredes Iacobi Iuntae (RFO 230 THO.ss. 1562, BN).

- (1570-71), Opera omnia, gratïs privilegïsque Pii V. ... typis excusa, Romae: ap. haeredes A. Bladii et J.O. Liliotum socios, ap. J. Accoltum (G189.4 THO.1, BN).

- (1570), Ex universa svmma sacrae theologiae doctoris vere angelici $S$. Thomae Aquinatis, dusumptae conclvsiones, Lovanii, Apud Ionnem Foulerum Anglum (RFO 93- 37925 RFO 93-37964, BN).

- (1590), Svmmae sacrae theologiae: in tres partes divisa, Bergomi, typis Comini Venturae (RFO 230 THO.s. 1590, BN).

\section{Facultad de Medicina}

Título 13, "De lo que a de leer el catedrático de prima de medicina. El primer año $[\ldots]$ el titulo $D e$ clementiz, Temperamentis, Umoriv, De pulsibus et urinis. El segundo año leerá de Diferencis febrium, De sanguinis micionem et De arte curativa/ad Glauconem. El tersero año leerá del Pidemias o del Método de Galeno. El catedrático de vísperas de medicina a de leer [...] Aforismos de Hipócrates comentados por Galeno..." (Proyecto..., 1991). Menciona las lecturas del segundo al cuarto año y para el "tersero" señala leer "del Pidemias o del Método de Galeno [...] El catedrático de vísperas de medicina a de leer lo ciguientte [...] El primer año leerá, de los siete libros de Yppócrates de Aforismos, los que fueren mas necesarios [...] El quarto año leerá los Pronósticos en algunos aforismos que convienen con los dichos pronósticos".
La obra más antigua de Galeno se conserva en la Biblioteca Histórica Doctor Nicolás León de la Facultad de Medicina de la UNAM: Galeni Librorum, 5 ed, Venetiis, Iunta, 1576, 6 vols.

De Hipócrates resguarda seis ediciones, las más antiguas son la de 1546 y la de 1596 que trata de los pronósticos:

- (1546), Hippocratis coi; medicorum omniumilonge principis opera quac ad nos estantumnia, Basileae, M. Adannos.

- (1596), Hippocratis prognosticum, in quo omnes divini viri tam genuinae $\mathcal{E}^{2}$ magnae tabellae quam spunie apocrypne..., Matriti, I. Iuntam.

La Biblioteca Nacional cuenta con seis impresos de Hipócrates, de 1552 a 1770, un ejemplo:

- (1552), Liber prognosticorvm Hippocratis Coi.nuper e greco in latinum sermonem translatus, cum praeclaris expositionibus additis, annotationibus in Galeni commentaries..., 2 ed., Salmanticae, Exudebat Andreas a Portonarijs (rfo 93-32665 rfo 94-411109, BN).

Algunos comentarios sobre las obras de Hipócrates:

- Francisco de Valles [1524-1592] (1567), Commentaria in prognosticum Hippocratis, Complvti, Apud Andream de Angulo (RFO 616 VAL.c.1517, BN)

- Francisco de Valles [1524-1592] (1561), In aphorismos \& libellum de Hippocratis commentaria, Compluti, Andreae ab Angulo (RFO 610 VAL.a.1561, BN)

- Bernardino Genga [1620-1690] (1744), Cirugia de hippocrates, y comentarios sobre sus aphorismos pertenecientes a la cirugía..., Madrid, Imprenta de Lorenzo Francisco Mojados (RFO 617 GEN.i.1744, BN) 
Título 14. "De lo que a de leer el catedrático de sirugia i anatomía", señala que los primeros dos años se leerán los libros de Galeno y los otros dos años la "sirujía meramente siguiendo a Guido, antiguo docto barón que se fundó con dotrina de Galeno y de Ippócrates y Avizena, y siga su moda en leer por capítulos" (ibid.).

En la Biblioteca Nacional se encuentra un interesante texto:

- Alonso Lopez Cornejo (1699), Galeno ilustrado, Avicena explicado, y doctores sevillanos defendisos: refútase la nveva con la anigua medicina, y manifiestase, que ni Hipocrates, Galeno Avicena, ni los Practicos antiguos ignóralo lo mas moderno, y que de ellos se ha deducido, y trasladado lo mas vtil. Dase a entender cuan pernicioso es regularmente usar de los Medicmentos Espagiricos, o Chimicos, y especialmente minerales, y antimoniales. Prvebase con antigvos, y modernos, que el método de los Doctores sevillanos es el mas vtil, y seguro en la curación de las tercianas assi exquisitas como notas, Sevilla, Juan de Puerta (RFO 610 LOP.g., BN)

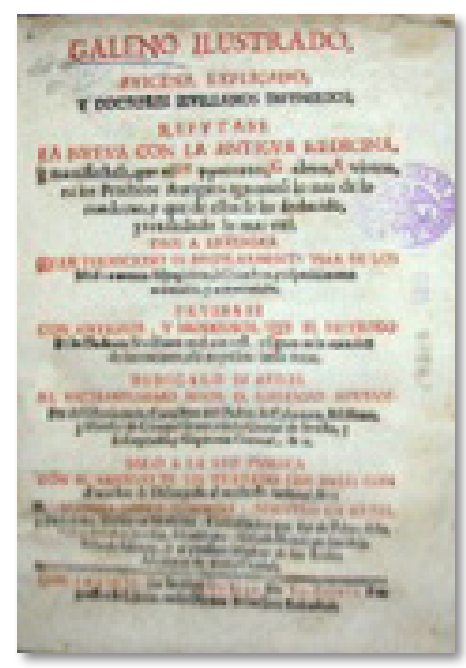

Alonso López Cornejo, Galeno ilustrados, 1699
De las obras de Guido en la Biblioteca Nacional se pueden mencionar:

- Guy de Chauliac [1300-1368] (1559), Gvidonis de Cavliaco in arte medica exercitatissimichirurgia nunc rerum non mediocri studio atque diligentia a pluribus mendis purgata, Lvgduni, Apud Sebastianum de Honoratis (FO617 GUY.i.1559, BN).

- Guy de Chauliac [1300-1368] (1596), Ars chirurgica, traducida al español e impresa en Valencia en 1596: Cirugia de Guido de Cauliaco (RFO 617 GUY.i.1559, BN).

\section{Cátedras sueltas}

Además de los cursos de las facultades mencionadas, se impartieron cátedras sueltas como astronomía, matemáticas, lenguas indígenas —náhuatl y otomí - aritmética, álgebra, geometría, etcétera, como ejemplo de los textos utilizados, el Arte de la lengua Mexicana y Castellana, de Alonso de Molina, publicado por Pedro Ocharte en 1571 y por Pedro Balli en 1576, fue lectura obligatoria para la cátedra de lengua mexicana en el siglo XVII, según se menciona en los Estatutos de Cerralvo, Título 39: De la cátedra de lengua mexicana 3. "Yten, se hordena que el que saliere con la cátedra, lea el Arte de la lengua mexicana por el de Molina, procurando mas enseñar ministros que hacer demostración de sientífico en ella" (Proyecto..., 1991:173).

José Ignacio Bartolache fue doctor en medicina y maestro suplente de matemáticas, nunca ganó la titularidad de la cátedra, pero fue autor del texto obligatorio de matemáticas: Lecciones matemáticas. Que en la real Universidad de México dictaba D. Josef Ignacio Bartolache... en la Imprenta de la Biblioteca Mexicana, 1769. Bartolache editó el primer periódico de noticias médicas, el Mercurio Volante, se puede decir que éste fue el primer periódico académico. 

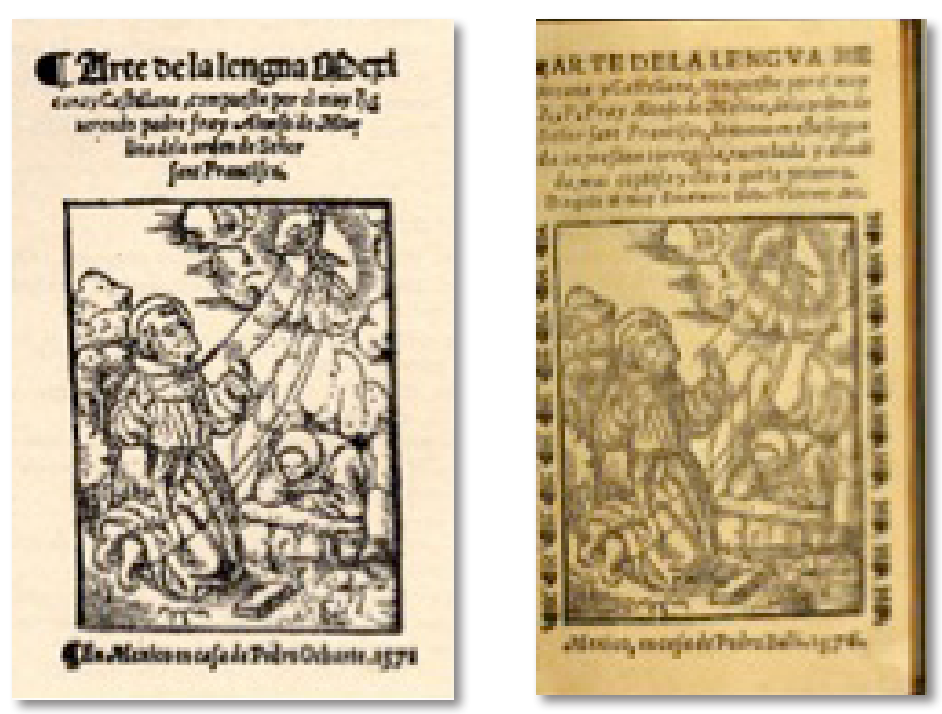

Alonso de Molina,

1510-1578

Arte de la lengua Mexicana y Castellana, 1571 y $1576^{2}$

\section{LECCMNES MATEMATTCA, QUE EN LAREAL UNTERTIOAO De Marico

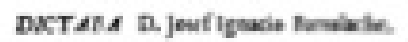 PRIMER QUADERNO, DEDKA $A D O$ AL. ECELENTIRMO fraOR DON CARLOS VRA NCECO DE CROIX,

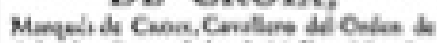

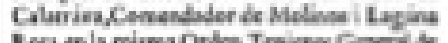

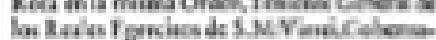

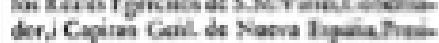 deres de la R. Aufencia de Mrecentuctue.

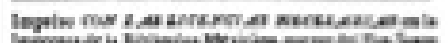

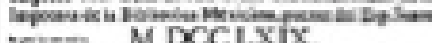

José Ignacio Bartolache, 1739-1790 Lecciones matematicas, 1769
TÍtulo 23. De los grados de licenciados, doctores y maestros. En el punto 9 se señalan los libros que el que se va a graduar debe escoger para su primera lección; se mencionan nuevamente, de acuerdo con la especialidad, los Decretales, el Digesto viejo, los libros del Maestro de las sentencias, las lecciones de Galeno, los Pronosticos, las lecciones de Avisena, de Aristoteles los ocho libros de los Fisicos y la Logica y el libro de las Oraciones de Cicerón: Marco Tulio Cicerón. M.T. (1603), Ciceronis Oratoinvm: a freigio notis perpetvis illustrtarum, Hanoviae, Impensis Claudij Marnij (805 CIC.or6 1603, BN).

Para las "oposisiones" mandaba que la "universidad tenga los libros de Derecho Canonico y Sivil, Maestro de las sentencias, y los demás libros de medicina y artes; y se guarden y se enquadernen y rrenueven de suerte que no pueda aver fraude. Y si no los ubiere se compren del dinero del arca de la universidad..." (ibid.: 111).

Los libros antes mencionados aparecen también en las Constituciones de la Real y Pontificia Universidad de México, Segunda edición, dedicada al rey nuestro señor Don Carlos III, México, Imprenta de D. Felipe de Zúñiga y Ontiveros, 1775, conocidas como Constituciones de Palafox. Para un ejemplo véase la Constitución GC.LXXV (p. 160), en la que se indica que para el examen de Teología se han de señalar puntos en el libro de las Sentencias, para la facultad de Cánones en las Decretales, para leyes en el Digesto viejo, para medicina en los Aforismos de Hipócrates entre otros.

\footnotetext{
${ }^{2}$ Imágenes tomadas del libro Impresos mexicanos del siglo XVI... (Fernández, 2009).
} 


\section{Formación de ingenieros en México}

El Colegio de Minería fue solicitado al rey por Joaquín Velázquez Cárdenas de León y Juan Lucas de Lassagna. Pidieron la fundación de un colegio que formara expertos en ciencias básicas relacionadas con esas actividades como las matemáticas, física, química y mineralogía. Fundado en 1792 por Fausto de Elhuyar (1755-1833), eminente mineralogista, comenzó con un programa académico semejante al propuesto por los antes mencionados, pero se negó a aceptar maestros novohispanos como Antonio León y Gama para el curso de física; Elhuyar prefirió contratar maestros europeos. El Colegio abrió sus puertas el 2 de enero de 1792 con la Carrera de Perito Facultativo de Minas, para lo cual los estudiantes debían cubrir cuatro años de cursos teóricos prácticos: matemáticas, física, química y mineralogía y dos más de prácticas en los reales de minas.

Durante estos años el Colegio tomó el liderazgo en la Colonia como institución educativa científica. En poco tiempo mostraba frutos sólidos, como la primera traducción al español de los Elementos de química de Lavoisier; la traducción de las Tablas mineralógicas de Karsten; el descubrimiento de Andrés Manuel del Río (catedrático de mineralogía) de un nuevo elemento (actualmente conocido como el Vanadio [...] se dieron a conocer nuevos minerales, se publicaron obras científicas como el Manual de Orictognosia de Manuel del Río y su Geometría subterránea, los Principios de Física Matemática y Experimental de F. Antonio de Bataller y el Tratado de amalgamación de la Nueva España de Federico Sonneschmidt (Ramos, 2007).

Este auge se vio interrumpido por los movimientos de Independencia y durante el siglo XIX vivió nuevos problemas por la lucha de conservadores y liberales.

De los libros mencionados por María de la Paz Ramos, se encuentran en la Biblioteca Nacional:
- Manuel del Río [1765-1849] (1795-1805), Elementos de orictognosia, ó, Del conocimiento de los fósiles, dispuestos según los principios de A.G. Werner, para uso del Real Seminario de Minería de México, México, Mariano Joseph de Zúñiga y Ontiveros, 2 vs. (G560.17 RIO.e. RSM 1795, BN).

- Antoine Laurent Lavoissier [1743-1794] (1797), Tratado elemental de chimica: dispuesto en un orden nuevo según los descubrimientos modernos, escrito en francés por M. Lavoissier, y traducido al Castellano para el uso del Real Seminario de Minería de Mexico, Mexico, Mariano de Zúñiga y Ontiveros, 2 vs. (RSM 1797 MALAV, BN).

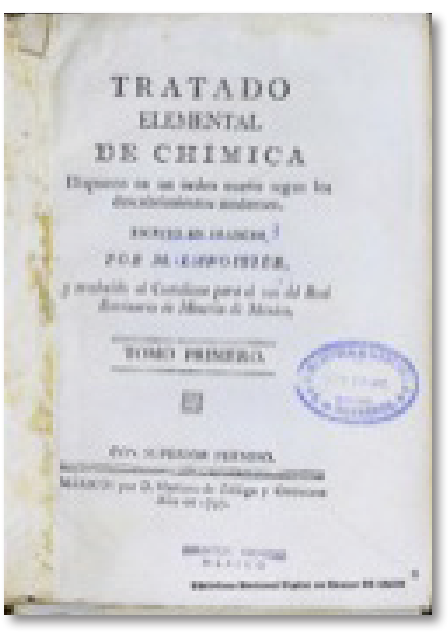

Antoine Laurent Lavoissier 1743-1794

Tratado elemental de chimica, 1797

- Dietrich Ludwig Gustav Karsten [1768-1810] (1804), Tablas mineralógicas dispuestas según siguen los descubrimientos mas recientes e ilustradas con notas, Traducida al castellano para el uso del Real Seminario de Minería por Andrés Manuel del Río, 3 ed. Alemana de 1800, México, Zúñiga y Ontiveros (Fondo San Carlos, BN).

- Friedrich Traugott Sonnesschmidt [1763-1824] (1825), Tratado de la amalgamación de Nueva España, sacado a luz por J.M.F., Paris, Galería de Bossange (G 67371 SON.t, BN). 


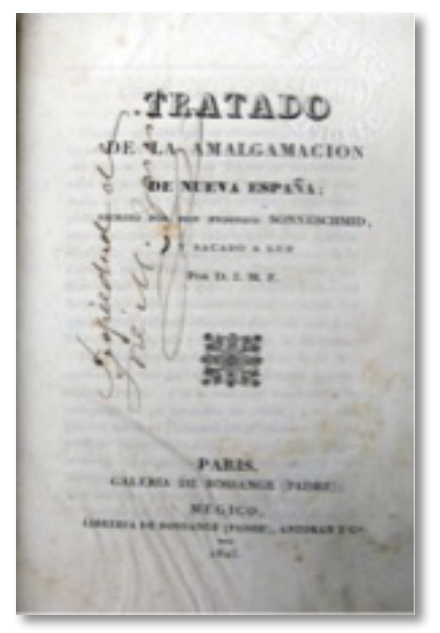

Friedrich Traugott Sonnesschmidt 1763-1824

Tratado de amalgamación de Nueva España, 1825

En La Biblioteca Nacional de España hay ejemplares pero sin año de impresión.

\section{Formación de arquitectos, pintores, escultores y grabadores. La Rel Academia de San Carlos de las Tres Nobles Artes}

Según Eduardo Báez Macías (2015: 9) la Academia de la Nueva España pasó por tres etapas: Escuela de Grabado (1778-1781), Escuela Provisional de Bellas Artes (1781-1783) y Real Academia a partir de 1783". En diciembre de 1783 el rey de España, Carlos III, aprobó la fundación de la Real Academia de San Carlos de las Tres Nobles Artes de la Nueva España a petición de Jerónimo Antonio Gil (17321798), tallador de la Casa de Moneda, con el fin de mejorar el grabado de las monedas. En la Academia se formaban principalmente arquitectos, pintores, escultores y grabadores siguiendo el modelo de la Real Academia de San Fernando de Madrid. Fue director de la Academia el famoso escultor Manuel Tolsá.

Dice Eduardo Báez (2015: 22):

los libros que venían para la Academia llegaban con la lozanía del pensamiento de la Ilustración y su particular incidencia en la Historia, las Ciencias

y la teoría del arte. Me basta con citar el Vitruvio de Ortiz y Sanz, la Conjuración de Catilina, mejor conocido como "el Salustio", el Quijote de Joaquín de Ibarra y el Tratado de las proporciones del cuerpo humano de Gerard Audran.

Entre los muchos textos utilizados se encuentran los de Andrea Palladio, del que citamos dos obras, y Pollio Vitruvio, de quien existen ocho obras, de 1497 a 1825, seis de las cuales pertenecieron a la Academia de San Carlos, una a los jesuitas y un incunable que fue propiedad de Sigüenza y Góngora.

- Andrea Palladio [1508-1580] (1581), I quattro libri dell architettura, Venetia, Appresso Bartolomeo Carampello (R722.7 PAL.q.1581, BN (Foto Felipe Cuenca)).

- Andrea Palladio [1508-1580] (1818), Studio elementare degli ordini di architettura, publicato da Giovan Battista Berti. Milano, Presso Batelli et Fanfani (BN, RFO 94-42812).

- Pollio Vitruvio (1497, 3 de agosto), De architectura libri $X$, Venetiis, per Simonem Papiensem dictum Bauilaquam (Incunable (Foto Felipe Cuenca), BN).

- Pollio Vitruvio (1556), I dieci libri dell'architetura: con due tauole, l'una di tutto quello si contiene per $i$ capi nell'opera, l'altra per dechiaratione di tutte le cose d'importanza, Di Vitruvvio, tr. et commentati da monsignor Barbaro Eletto patriarca dá Qvileggia, Vinegia, Franceso Marcolini (724F VIT.d.1556, BN).

- Pollio Vitruvio (1567), De architectura libri decem, com commentariis Danielis Barbari, electipatriarchae aquileiensis: moltis aedificiorom, horologiorvm, et $\mathrm{ma}^{-}$ chinarvm descriptionibrs, $\tilde{n}$ figuris, una cum indicibus copiosis, auctis $\tilde{n}$ illustratis, Venetiis, ap. Franciscium Senensem, ñ Ioan, Crugher Germanum (RFO 724 VIT.d. 1567, BN). 

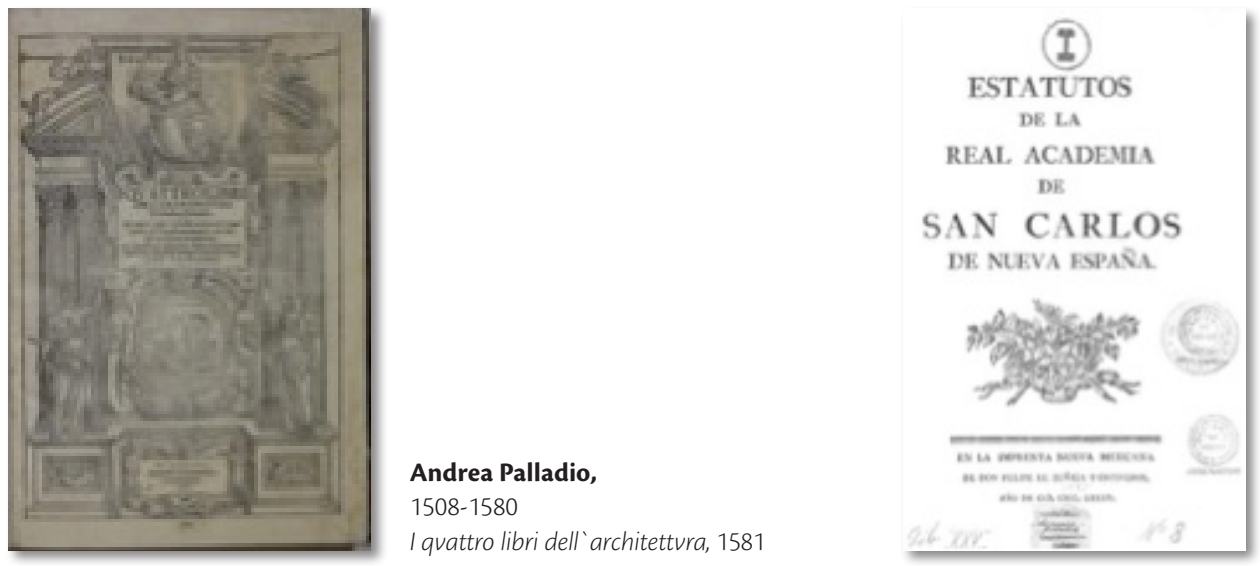

Estatutos de la Real Academia de

I quattro libri dell 'architettura, 1581
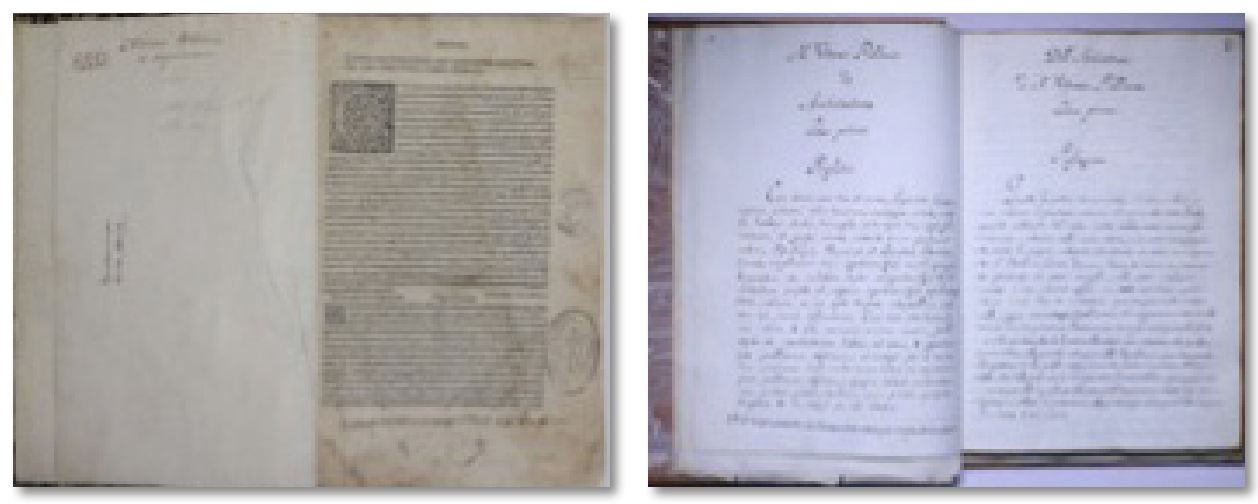

Pollio Vitruvius,

De Architectura libri x, 1497

- Pollio Vitruvius (ca. 1790), M. Vitruiri Pollionis de Architectura: Dell' architettura di M. Vitruvio Pollione, (Mss. EASC XI-93, BN (Foto Gisel Aguilar López)).

- Pollio Vitruvius (1787), Los diez libros de architectura, Tr. del latín y comentados por Joseph Ortiz y Sanz, Madrid, Imprenta Real (724 VIT.d. $1787, \mathrm{BN})$

Si bien no es un libro de texto, el siguiente es un bello libro académico:

- Estatutos de la Real Academia de San Carlos de Nueva España, México, Imprenta Nueva Mexicana de Don Felipe Zúñiga y Ontiveros, 1785, (1785 M4ACA XXV-8, BN).

\section{Los colegios jesuitas}

Como es conocido, los jesuitas seguían las disposiciones de la Ratio Studiorum de 1599 que combinaba la teoría con la práctica. "Los jesuitas no tenían las restricciones de las órdenes monásticas y estaban ligados de manera especial al Papa a través de un cuarto voto de obediencia directa a su persona; se encontraban también en trato con seglares e instituciones seculares, y desde el punto de vista filosófico estaban más cerca de santo Tomás de Aquino y de Aristóteles que de Platón y de san Agustín" (Zermeño, 2001: 87). Los textos de Francisco Suarez y Luis de Molina fueron obras muy consultadas. En su prefacio al Zodiaco Mariano Francisco Javier Lazcano, quien enseñaba la teología de Francisco Suárez en la Universidad de México declara (Brading, 2001: 66). 


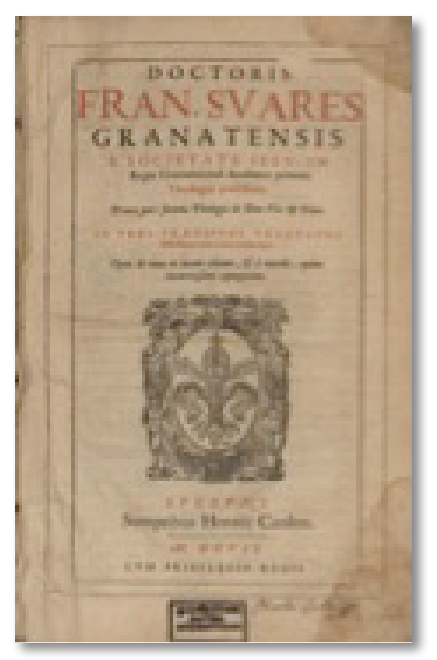

Francisco Suárez,

1548-1617

Summae theologia, 1607

- Francisco Suárez [1548-1617] (1607), Summae theologia de Deo vno \{et\} Trino, Fran. Svares, Lvgdvni, Sumptibus Horatii Cardon (RFO 230 SUAs 1607, BN (Foto Felipe Cuenca)).

- Francisco Suárez [1548-1617] (1613), Tractatus de legibus ac Deo legislatore in decem libros distributus, P.D. Francisco Svarez Grantensi, Atverpiae, Apud Ioannem Keerbergvm (RFO 340 SUA 1613, BN).

Por su parte Luis de Molina y sus famosas reflexiones filosóficas sobre "la teoría del justo precio" fue autor de Concordia liberi arbitriicum gratiae donis, divina praescientia, predestinatione et reprobatione", Lisboa, 1588.

Zermeño asienta que la posición de Molina también es parte de una reforma pedagógica que abarcó a todas las facultades europeas y que implicó la sustitución del Libro de las Sentencias del maestro Pedro Lombardo como texto único de enseñanza, por la Summa Theologica de Tomás de Aquino. La reforma fue impulsada desde Salamanca por el dominico Francisco de Vitoria (Zermeño, 2001: 84). Resultados de estas lecturas fueron los debates de jesuitas y dominicos sobre el probabilismo.
Algunos de los jesuitas novohispanos que produjeron libros de texto sobre la Scientia media fueron Antonio Núñez de Miranda, 1669; Diego Marín de Alcázar, 1690; José Pedro Cesati, 1674; Pablo Salceda, 1671 y Matías Blanco, 1708.

\section{Otras opiniones interesantes}

Ignacio Osorio señalaba que la obra de Andrea Alciato, Omnia Domini Andreae Alciati Emblemata, impresa en México por Antonio Ricardo en 1577, formó parte del programa educativo de los jesuitas mexicanos desde el siglo XVI y observaba que los jesuitas consideraban a los epigramas llenos de erudición humanista y excelente para la formación literaria de los estudiantes (Fernández, 2009: 279).

La Biblioteca Nacional cuenta con varias ediciones de los emblemas de Alciato, pero no con la impresa en México, dos ejemplos:

- Andrea Alciati [1492-1550] (1589), Omnia Andreae Alciati V. C. Emblemata: cum commentariis, quibus emblematum aperta origine mens auctoris explicantur, E obscura omnia, duabia[m]que illustrantur, Parisiis, Ap Ioannem Richeriu[m] (RFO 704.946.ALC.o.1589, BN).

- Andrea Alciati [1492-1550] (1591), Emblemata, Lvgdvni Batavorum, Ex oficina Plantiniana, Raphelengium (RFO 704.946 ALC.e.1591, BN)

José Gallegos Rocafull opinaba que Fray Bartolomé de Ledesma, autor de Septem Novae Legis Sacramentis Summarium, impresa en México por Antonio de Espinosa en 1556, escrita por compromiso, era de un estilo pesado y monótono, compuso otras que se perdieron, y que según García Icazbalceta perdura aún "la honorífica memoria de sus tratados de fustitia et jure, que acudían a oir y escribir en la Universidad de México los estudiantes cursantes de las cátedras de Leyes" (Gallegos, 1951: 233). 
Por su parte, José Pascual Buxó, al referirse a los impresos mexicanos del siglo XVII, menciona:

De las imprentas novohispanas salían en competencia numerosos libros de los que hoy llamamos 'de texto'. Así se publicaron para utilidad de los estudiantes: la Construccion y explicacion de las reglas del genero conforme al Arte de Antonio, de Diego López (Viuda de Calderón, 1660) y la Explicacion del Libro Quarto, Conforme a las reglas del Arte de Antonio de Nebrija (Rodriguez Lupercio, 1664). También en Puebla, Fernández de León publicó en 1685 Explicacion de los Libros Quarto y Quinto de la Grammatica conforme al Arte de Antonio de Nebrija, para los estudiantes de los Colegios de San Juan y San Pedro. Para la cátedra de retórica "eran indispensables los florilegios de poesía latina" como el Thesaurus Poetarum. In gratiam inventutis Poetices studiose defossus, de Pedro de Salas. (México, Pedro Robledo, 1641) (Pascual, 2001: 66-67).

Para terminar deseo recordar a ese gran novohispano, o sería más acertado decir a ese gran mexicano, Juan José de Eguiara y Eguren y su valiosa opinión sobre textos que debían leer los alumnos. Eguiara y Eguren se recibió de bachiller en Teología leyendo una hora de ampolleta sobre el maestro de las Sentencias, Pedro Lombardo, el 29 de abril de 1712, fue el autor de la primera bibliografía impresa en América en 1755, la Bibliotheca Mexicana y fue maestro de filosofia en la Real Universidad. Eguiara y Egurén, en cuanto al método docente que se seguía en la Universidad, mencionó que:

exceptuando a los carmelitas descalzos que estudian el curso de filosofia obligatorio entre los de su orden, publicados por sus colegas de la Universidad de Alcalá, todos los demás alumnos de cualquiera escuela u orden religiosa, universidad o colegio, no solo de México, sino de cualquier centro de enseñanza de la América Septentrional, siguen las lecciones que sus maestros les dictan desde la cátedra y anotan en sus cuadernos los cursos elaborados para su auditorio por cada uno de aquellos. Testimonio de lo que decimos darán lo numerosos cursos manuscritos de que en nuestra $B I^{-}$ BLIOTHECA hablaremos aunque omitiendo muchísimos por lo trabajoso y pesado que resultaría averiguar sus autores y nombrarlos a todos (Millares, 1957: 50).

O sea que no era fácil identificar los títulos de los textos que debían leer los estudiantes, como sucede con los mencionados en los estatutos de la Universidad y que se relacionan en este capítulo.

Hasta aquí este acercamiento a los maravillosos libros académicos de la ilustre Real y Pontificia Universidad de México y otras instituciones educativas en la Nueva España. 


\section{Referencias}

Báez, Eduardo (2015), "Presentación”, en Silvia Salgado y Gisselle Aguilar (coords.), La Biblioteca de la Academia de San Carlos en México, México, IIBI/Facultad de Artes y Diseño/DGAPA, UNAM, p. 9.

Beuchot, Mauricio (1991), Estudios de historia y de filosofia en el México colonial, México, UNAM-IIB.

Beuchot, Mauricio (1987), Filósofos dominicos novohispanos (entre sus colegios y la Universidad), México, UNAM, CESU (La Real Universidad de México Estudios y textos, II).

Brading, David (2001), "La patria criolla y la Compañía de Jesús”, Artes de México, núm. 58, pp. 59-70.

Fernández de Zamora, Rosa María (2009), Los impresos mexicanos del siglo XVI: su presencia en el patrimonio cultural del nuevo siglo, México, UNAM, CUIB.

Gallegos Rocafull, José M. (1951), El pensamiento mexicano en los siglos XVI y XVII, México, Centro de Estudios Filosóficos.

Los colegios jesuitas en la Nueva España (2001), Artes de México, núm. 58.

Millares Carlo, Agustín (1957), Don Fuan Fosé de Eguiara y Eguren (1695-1763) y su Bibliotheca Mexicana, México, UNAM.

Pascual Buxó, José (2001), "Impresos e impresores mexicanos novohispanos del siglo XVII", en Memoria de México y el Mundo, México, UNAM, Dirección General de Publicaciones y Fomento Editorial, pp. 47-75.
Pérez Sanvicente, Guadalupe (1970), "Las cédulas de fundación de las universidades de México y de Lima (Ensayo de interpretación)", Estudios de Historia Novohispana, vol. 3, pp. 1-23.

Proyecto de estatutos ordenados por el virrey Cerralvo (1991), Edición Crítica de Enrique González, México, UNAM, Centro de Estudios sobre la Universidad (Real Universidad de México Estudios y Textos, 3).

Ramírez González, Clara Inés y Mónica Hidalgo Pego (2001), "Los saberes universitarios", en La Universidad de México. Un recorrido histórico de la época colonial al presente, México, UNAM, CESU, p. 73.

Ramos Lara, María de la Paz (2007), "El Colegio de Minería. La Escuela Nacional de Ingenieros y su proyección en otras instituciones educativas de la ciudad de México (siglo XIX)", en María de la Paz Ramos Lara y Rigoberto Rodríguez Benítez (coords.), Formación de ingenieros en el México del siglo XIX, México, UNAM-CIICH/UAS- Facultad de Historia, 2007.

Sánchez Menchero, Mauricio (2012), El corazón de los libros. Alzate y Bartolache, lectores y escritores novohispanos (Siglo XVIII), México, UNAM, CIICH (colección Debate y reflexión).

Zermeño P. Guillermo (2001), "La filosofía jesuita novohispana en perspectiva", Artes de México, núm. 58, pp. 79-88.

\section{Cómo citar este artículo:}

Fernández-de-Zamora, Rosa-María (2021), "Libros académicos en la Nueva España”, Revista Iberoamericana de Educación Superior (RIES), vol. XII, núm. 34, pp. 222-238, DOI: https://doi.org/10.22201/iisue.20072872e.2021.34.989 [Consulta: fecha de última consulta]. 